\title{
Structural and Band Structure Investigation of Iron Oxide Nanoparticles Incorporated PVA Nanocomposite Films
}

\author{
Muhammad Aslam ( $\sim$ aslamfaridipk@gmail.com ) \\ National Textile University https://orcid.org/0000-0002-2447-4652 \\ Muhammad Basit \\ National Textile University \\ Muhammad Ahmad \\ National Textile University \\ Zulfiqar Ali Raza \\ National Textile University
}

\section{Research Article}

Keywords: Iron oxide, Nanocomposite, Optoelectronics, Polyvinyl alcohol, PVA

Posted Date: December 16th, 2021

DOI: https://doi.org/10.21203/rs.3.rs-1166776/v1

License: (c) (i) This work is licensed under a Creative Commons Attribution 4.0 International License. Read Full License 


\section{Abstract}

The work reported here deals with the fabrication and characterization of iron oxide $\left(\mathrm{Fe}_{3} \mathrm{O}_{4}\right)$ nanoparticles (NPs - IONPs) incorporated polyvinyl alcohol (PVA)-based nanocomposite films. The nanocomposite films, fabricated via solution casting route, have been characterized using advanced analytical techniques including x-ray diffraction (XRD), scanning electron microscopy (SEM), energy dispersive spectroscopy (EDS), and UV-visible (vis.) spectroscopy. There observed notable changes in the structural phases, crystallite size ( 2.3 to $2.1 \mathrm{~nm}$ ), d-spacing ( 0.131 to $0.134 \AA$ ) , optical absorption edge (5.12 to 4.84 $\mathrm{eV}$ ), indirect bandgap (4.99 to $4.68 \mathrm{eV}$ ), direct bandgap (5.35 to $5.20 \mathrm{eV}$ ), and band tail ( 0.57 to $0.89 \mathrm{eV}$ ) from native PVA to nanocomposite films. The refractive index and optical conductivity enhancements were also observed on incorporating IONPs into the PVA matrix. It could be inferred that a minute loading of IONPs might induce significant alternation in opto-structural properties of the PVA-based nanocomposites for potential optoelectronic applications.

\section{Introduction}

Polymer-based nanocomposites have extensively been considered for various applications in science and technology including microelectronics, optoelectronics, sensing, fuel cells, energy storage, electromagnetic shielding, and so on [1-3]. Inorganic nanofillers can induce extraordinary attributes into polymeric composites including improved structural, optical, mechanical, electrical, and thermal properties [4]. Likewise, iron oxide $\left(\mathrm{Fe}_{3} \mathrm{O}_{4}\right.$, magnetite) nanoparticles (NPs - IONPs) provide a wonderful platform for tuning the said properties of polymer based composites for optoelectronic applications. The IONPs express poor wettability and acquire agglomeration tendency in the dispersion due to their magnetic essence [1] which may hinder their dispersibility in the aqueous media. This could be compensated by coating their surfaces with some hydrophilic/polar polymeric matrices like PVA.

The PVA, due to their hydroxyl moieties, could provide surface coverage to the IONPs making them somehow hydrophilic composite with good transparency and other optical properties. The PVA is additionally, easily processable, biodegradable, and biocompatible polymer [2, 5]. The PVA has been extensively used as base matrix for fabrication of polymer nanocomposites. Abdullah et al. [6] prepared PVA/aluminum iodide nanocomposite films using solvent evaporation approach. Mohapatra et al. [7] reported phosphate functionalized PVA composite incorporated with iron NPs. Chastellain et al. [8] fabricated PVA-loaded magnetite NPs using the co-precipitation route. Guo et al. [9] prepared PVA based a-phase iron oxide NPs composites. Selvi et al. [10] prepared cadmium oxide/PVA nanocomposites via the solution casting technique.

Herein, a green route of solution casting has been acquired to fabricate IONPs mediated PVA nanocomposites followed by $x$-rays diffraction (XRD) for crystal structure, scanning electron microscopy (SEM) for surface morphology, energy dispersive spectroscopy (EDS) for elemental analysis, Fourier transform infrared (FTIR) spectroscopy for any changes in the chemical functionalities, UV-visible (UVvis) spectroscopy for optical parameters of the prepared polymer films. 


\section{Materials And Methods}

\section{Materials}

PVA granules (av. mol. mass 47,000 $\mathrm{g} \mathrm{mol}^{-1}, 70-90 \%$ hydrolyzed) and IONPs of av. z-average $<50 \mathrm{~nm}$ were purchased from Sigma Aldrich. Deionized water (18 $\mathrm{M} \Omega)$ was obtained from Millipore.

\section{Fabrication of IONPs mediated PVA nanocomposites}

The IONPs mediated PVA nanocomposite films were fabricated through an easy and green route of solution casting [11]. The PVA granules $(2 \mathrm{~g})$ were dissolved in the deionized water under constant magnetic stirring at $300 \mathrm{rpm}$ and $60^{\circ} \mathrm{C}$ for $3 \mathrm{~h}$. The IONPs (as $0.00,0.25,0.50$, and $1.00 \mathrm{wt} \%$ ) were dispersed separately in the deionized water under ultrasonication at $30^{\circ} \mathrm{C}$ for $1 \mathrm{~h}$. Both contents were magnetic stirred at $300 \mathrm{rpm}$ and $60^{\circ} \mathrm{C}$ for $1 \mathrm{~h}$ followed by ultrasonication for $1 \mathrm{~h}$ to make a homogenized blend, then poured into a clean Petri dish for oven drying at $30^{\circ} \mathrm{C}$. The dried nanocomposite films were peeled off and analyzed, accordingly. The average thickness of the prepared films was calculated as $160 \pm 10 \mu \mathrm{m}$ under the ambient conditions.

\section{Characterization}

XRD analysis of the prepared samples was done on an X'Pert PANalytical device in the $2 \theta$ scanning range of $10-80^{\circ}$. The surface morphology of the developed films was studied under a SEM (Quant 250, FEI) installed with an EDS detector operated at a potential difference of $45 \mathrm{kV}$. Fourier transform infrared spectroscopy (Bruker Tensor 27) was done to indicate changes in any functional group in the composite films in the wavenumber scanning range of $600-4,000 \mathrm{~cm}^{-1}$. The PVA-based films were analyzed for UVvis. absorption on a UV-vis spectrophotometer (UV-2800, Biotechnology Medical Services, USA) in the scanning range of $200-1000 \mathrm{~nm}$.

\section{Results And Discussion}

\section{XRD analysis}

Figure 1 represents XRD scans of PVA and IONPs incorporated PVA nanocomposites. A broad peak at $2 \theta$ of $19.4^{\circ}$ was observed having a crystal plane (101) in the case of pure PVA film (Fig. 1a) indicating its semicrystalline nature $[10,11]$. The literature shows the XRD diffraction peaks of IONPs (Fig. 1b) at $2 \theta$ positions of $30.35^{\circ}(220), 35.81^{\circ}(311), 43.39^{\circ}(400), 53.88^{\circ}(422), 57.39^{\circ}(511)$, and $63.09^{\circ}(440)$ with cubic crystal structure and Fd-3m space group (JCPDS card 88-0315) [Mohan Lal]. Figure 1c-1e shows that the width of the said diffraction peak (at $19.4^{\circ}$ ) slightly shifted toward a higher diffraction angle after incorporation of IONPs in the prepared films. The d-spacing of pure PVA film was measured as $0.131 \AA$ employing Bragg's relation [5]; whereas, at 1 wt\% IONPs loading in the PVA matrix, the d-spacing slightly increased to $0.134 \AA$. The crystallite size of pure PVA film was calculated as $2.3 \mathrm{~nm}$ using the Debye Scherrer's relation [12] which decreases to $2.1 \mathrm{~nm}$ at $1 \mathrm{wt} \%$ IONPs loading in the PVA based films. All 
these observations indicate structural changes in the nanocomposite films after incorporation of IONPs. The microstrain and dislocation density might also be increased. The hydroxyl groups in the PVA matrix behave as chelating centers (where metal oxides are bonded) for the IONPs leading to the formation of nanocomposites. The XRD figure shows that the $\mathrm{Fe}_{3} \mathrm{O}_{4} \mathrm{NPs}$ peaks were merged into the noise due to their smaller incorporation ( 0.25 to $1 \mathrm{wt} \%$ ) in PVA matrix and causes an amorphous look to the prepared nanocomposites [10].

\section{Surface morphology analysis}

Figure 2 shows SEM micrographs of both native and IONPs loaded PVA films with their respective EDX elemental analysis results. Figure 2a shows that the native PVA films were uniform without any air bubbles or foreign particles indicating their phase purity. Figure $2 \mathrm{~b}$ indicates a good distribution of IONPs in the PVA matrix with some aggregations which might be due to large surface energy and magnetic interactions between the nanocomposite crystallites. The overall distribution of IONPs might be attributed to their restrictive mobility in the viscous polymeric environment and chain entanglement. Likewise, there observed no voids or cracks on the resultant nanocomposite films. This might be due to strong hydrogen bonding between IONPs and the hydroxyl groups of the PVA chains thus producing the crack-free nanocomposite.

Figure 2 indicates the EDS patterns of the prepared samples. Carbon (66.6\%) and oxygen (33.4\%) elements were found in pure PVA sample whereas, carbon (62.1\%), oxygen (37.1\%) and iron $(0.2 \%)$ elements were found in IONPs loaded PVA sample. No other element was found in the EDS patterns, verifying the purity of the developed samples.

\section{FTIR analysis}

The FTIR spectra showed the surface chemical interaction between IONPs and the PVA matrix in the nanocomposite (Fig. 3). There observed two peaks at 2854 and $2924 \mathrm{~cm}^{-1}$ due to C-H asymmetric and symmetric vibrations, respectively [5]. An OH-stretching peak was found both in the native PVA and the IONPs mediate PVA films at $3276 \mathrm{~cm}^{-1}$ [12]. This indicated the hydrophilic nature of PVA films in native and also after nanocomposite formation. A peak at $1648 \mathrm{~cm}^{-1}$ indicated moisture absorption on the composite surface under ambient conditions which indicates that the PVA retained its hydrophilic behavior. A peak at $1089 \mathrm{~cm}^{-1}$ was attributed to $\mathrm{C}-\mathrm{O}$ acetyl stretching vibration in the PVA matrix [13]. There observed two peaks at 836 and $1425 \mathrm{~cm}^{-1}$ because of $\mathrm{CH}_{2}$ rocking and $\mathrm{C}-\mathrm{C}$ stretching, respectively [14]. There observed a slight variation in the fingerprint of the nanocomposite films as compared to the native PVA film; this confirms the good distribution of IONPs in the PVA-based nanocomposite [13].

\section{Optical absorption}

Figure 4 shows the optical absorption of native PVA and 0.25-1 wt\% IONPs loaded PVA films. There observed notable variation in the results of composite films in the form of absorption of electromagnetic radiations in the UV vis. regions after incorporating IONPs. The absorption in these two regions could be 
explained based on the molecular orbital theory, according to which the electrons are promoted from the ground energy level to the allowed higher energy levels with possible transitions of $\sigma$ to $\sigma^{\star}, n$ to $\pi^{\star}$, and $\pi$ to $\pi^{\star}$. The optical electronic transitions in the vis. region are mostly caused by impurities or defects in the test material [15]. Figure 4 shows two optical-electronic $\pi$ to $\pi^{*}$ and $n$ to $\pi^{\star}$ transitions which appear as absorption peaks at 427 and $278 \mathrm{~nm}$, respectively [5]. Various applications in optoelectronic devices depend on the electronic transitions in the vis. region. The results demonstrate that on rising the contents of IONPs in the PVA matrix, the absorption intensities in both transitions increased; in accordance with the literature $[10,15]$. It could also be noticed that absorbance was the highest in the UV region and the lowest (or minimum) in the vis. region of the electromagnetic spectrum.

\section{Optical Band gap}

The absorption coefficient $a$ is beneficial in predicting the variations in the optical band structure of polymer nanocomposites. The absorption coefficient was calculated using Eq. (1) from the absorbance data collected from UV-vis. scans [17].

$$
\alpha=2.303 \frac{A}{d}
$$

Where $A$ and $d$ are absorbance and sample thickness, respectively. Figure 5(a) indicates the absorption coefficient patterns of native PVA and IONPs loaded PVA films. The absorption coefficient values were obtained from the $a$ versus $h v$ curves, by extrapolating their linear portions to nil absorption. The absorption edge values were shifted to lower energy after incorporation of IONPs in the PVA matrix, owing to the charge-transfer complex formation in the resultant nanocomposites $[11,16]$. The a value decreased from $5.12 \mathrm{eV}$ (native PVA) to $4.84 \mathrm{eV}$ for $1 \mathrm{wt} \%$ IONPs loaded PVA film; this depicts decreasing trend in the optical bandgap of the developed nanocomposite film. Aziz et al. [18] calculated the a for both pure PVA and 5 wt\% aluminum-doped PVA composite being 5.25 and $4.26 \mathrm{eV}$, respectively.

Polymer nanocomposites with small bandgaps are usually required for optoelectronic or photonic devices. The bandgap Eg is a dominant parameter in the optical band structure that could be calculated by Tauc's plot using Eq. (2) $[11,16]$.

$\alpha h v=B\left(h v-E_{g}\right)^{x}$

Where $h, v$, and $B$ are the Planck's constant, the incident photon frequency, and the constant element, respectively. The factor $B$ is assumed as constant in the optical energy bands and it majorly relies on the transition probability of the energy bands. The index $x$ is associated with the density of states of electrons which defines the nature of an optical transition. In the optical transitions, it might have values of $1 / 2,3 / 2,2$, or 3 depending on nature of the transition [10]. Figure $5(b)$ indicates a graph of $(a h v)^{2}$ versus $h v$. The direct bandgap was obtained by extrapolation of the smooth part of the curve to the base value of $(a h v)^{2}$. It could be observed that the direct $E g$ for the pure PVA was $5.35 \mathrm{eV}$ which was reduced to 5.20 $\mathrm{eV}$ in the case of $1 \mathrm{wt} \%$ IONPs loaded PVA film. This transition takes place from the filled higher 
molecular orbital to empty lower molecular orbital [19]. Figure 5(c) shows the graphs between $(a h v)^{1 / 2}$ and $h v$. The indirect $E g$ for pure PVA film was calculated as $4.99 \mathrm{eV}$ which was reduced to $4.68 \mathrm{eV}$ with 1 wt\% IONPs loaded PVA film. The literature indicates that the indirect bandgap values are normally smaller than the direct bandgap values for polymer nanocomposites $[11,20]$.

The band tails of PVA and IONPs/PVA nanocomposites were measured using a plot $\ln (a)$ versus $h v$, by considering the slope of its linear part [19]. The band tail values for pure PVA, $0.5 \mathrm{wt} \%$ and $1 \mathrm{wt} \%$ IONPs incorporated PVA films were observed as $0.57,0.73$, and $0.89 \mathrm{eV}$, respectively. Abdullah et al. [21] observed a similar trend in band tail behavior after doping the PVA with aluminum iodide. Herein, incorporating IONPs in the PVA matrix, some defects and clusters might be introduced in the nanocomposite films; alongside, local energy states are produced in the forbidden energy band gap, Fermi level shifts, energy band gap narrows, and conductivity (optical as well as electrical) increases [18, $20,21]$. The enhancements in band tails followed Urbach empirical relation.

\section{Refractive index}

Refractive index $\mathrm{n}$ indicates the usability of a developed translucent composite for possible photonic and optoelectronic devices. Usually a high refractive index is desirable for such purposes [19]. It could be computed using the reflectance R data acquired from UV-vis. results, using Eq. (3) [11].

$$
n=\left(\frac{1+R}{1-R}\right)+\sqrt{\frac{4 R}{(1-R)^{2}}-k^{2}}
$$

The extinction coefficient $k$ could be measured using a relation: $k=\frac{\alpha \lambda}{4 \pi}$. Figure 6 (a) shows the plot of refractive indices versus wavelength for native PVA and IONPs/PVA nanocomposite films with various loadings. The figure shows that changes in refractive index values depend upon IONPs contents and the incident wavelengths. The effect of increasing NPs on the refractive index is more prominent in the UV region, comparatively. On increasing incident wavelengths, the refractive index decreases. The trend of figure $6(a)$ is in accordance with the literature $[17,19,22]$. Refractive indices in the UV region are much higher than the vis. region. Thus, the refractive indices might be tuned using $\mathrm{Fe}_{3} \mathrm{O}_{4} \mathrm{NPs}$ of various loadings not only in specific wavelength region but also at one particular wavelength. The changes in refractive indices of the prepared nanocomposites are due to addition of the $\mathrm{Fe}_{3} \mathrm{O}_{4} \mathrm{NPs}$ in the PVA matrix that have created localized states in forbidden energy bands regions. The curve trend of refractive indices versus wavelength suggests the use of IONPs/PVA nanocomposites in optical communications equipment $[19,21]$.

\section{Optical conductivity}

The optical conductivity $\sigma$ was calculated using $n$, a, and speed of light c, by Eq. (4) [17]:

$$
\sigma=\frac{n c \alpha}{4 \pi}
$$


Figure 6(b) shows a plot of optical conductivity versus wavelength for $\mathrm{Fe}_{3} \mathrm{O}_{4} / \mathrm{PVA}$ nanocomposite films at different loadings. The figure indicates that optical conductivity is higher in the UV region than in the vis. region, because of the greater absorption of incident photons by the IONPs [1]. It could be noted that in the vis. region, the optical conductivity of the prepared nanocomposite films slowly drops to constant values, approximately. It could also be noted that the optical conductivity depends upon IONPS incorporation, and the conductivity enhancing trend could be observed at each incident wavelength. The graph depicts the small optical conductivity in the vis. region that is due to smaller absorption of photons in this region and as a result smaller electron excitation. The same trend of optical conductivity versus wavelength for polymer nanocomposite films has been observed in the literature $[5,17,19,21,22]$. The excellent optical conductivity of IONPS/PVA nanocomposite films in the UV region makes them a suitable candidate for UV filter material.

Overall, the IONPs/PVA nanocomposite films have been synthesized successfully using solution casting approach. The XRD, SEM, and FTIR analyses indicate good dispersion of IONPs in the PVA matrix. On incorporating $1 \mathrm{wt} \%$ IONPs in PVA matrix, the following observations have been noted: crystallite size decreased from $2.3 \mathrm{~nm}$ to $2.1 \mathrm{~nm}$, interplanar distance increased from 0.131 to $0.134 \AA$, microstrain and dislocation density increased, optical absorbance in UV, as well as visible region, increased, absorption edge reduced from 5.12 to $4.84 \mathrm{eV}$, direct bandgap reduced from 5.35 to $5.20 \mathrm{eV}$, indirect band gap reduced from 4.99 to $4.68 \mathrm{eV}$, band tails enhanced from 0.57 to $0.89 \mathrm{eV}$, optical conductivity and refractive index both enhanced in the ultraviolet and visible region. Hence smaller incorporation of IONPS has made the fabricated nanocomposites unique.

\section{Conclusions}

The IONPs/PVA nanocomposite films have been fabricated through an easy and green route of solution casting approach. The crystallite size of PVA was detected minimized after incorporation of IONPs in the PVA matrix. Various investigations of XRD, SEM, EDX, and FTIR showed the presence of incorporated phases and good dispersion of NPs in the PVA matrix. The FTIR findings showed the bindings of IONPS with PVA and their coatings with PVA matrix. The incorporated NPs increased the cross-linking with clear optical property enhancements. Clear changes in the structural phase and optical properties comprising of optical band gap, Urbach energy, refractive index, and optical conductivity were observed when IONPs were incorporated into the PVA matrix. The enhancement in Urbach energy, reduction in the band gap region, and enhancing trend of optical conductivity by IONPs incorporation in PVA matrix suggested the formation of local energy states in the bandgap region of the fabricated films. Enhanced refractive index, improved optical as well as polarization attributes of the fabricated nanocomposites suggested their utilization in materials for optoelectronic devices.

\section{Declarations}

\section{Statements \& Declarations}




\section{Funding}

The authors declare that no funds, grants, or other support were received during the preparation of this manuscript.

\section{Financial Interest}

All contributing authors have no financial interest.

\section{Author Contributions}

All authors contributed to the study conception and design. Material preparation, data collection and analysis were performed by Muhammad Aslam, Muhammad Basit, Muhammad Ahmad, and Zulfiqar Ali Raza. The first draft of the manuscript was written by Muhammad Aslam and all authors commented on previous versions of the manuscript. All authors read and approved the final manuscript.

\section{References}

[1] M. Lal, S.R. Verma, Macromol. Symp. 376(1) 1700017 (2017)

[2] M. Aslam, M.A. Kalyar, Z.A. Raza, Polym. Eng. Sci., 58(12), 2119-2132 (2018)

[3] M. Aslam, M.A. Kalyar, Z.A. Raza, Polym Bull. 76(1); 73-86 (2019)

[4] M. Aslam M, Z.A. Raza, A. Siddique, Polym Bull. 78(4):1955-65 (2021)

[5] M. Aslam, M.A. Kalyar, Z.A. Raza, J. Electron. Mater. 47(7), 3912-3926 (2018)

[6] O.G. Abdullah, S.A. Hussen, Adv. Mater. Res. 383, 3257-3263, (2012)

[7] S. Mohapatra, N. Pramanik, S.K. Ghosh, P. Pramanik, J. Nanosci. Nanotechnol. 6(3):823-9 (2006)

[8] M. Chastellain, A. Petri, H. Hofmann, J. Colloid Interface Sci. 278(2):353-60 (2004)

[9] Z. Guo, D. Zhang, S. Wei, Z. Wang, A.B. Karki, Y. Li, P. Bernazzani, D.P. Young, J.A. Gomes, D.L. Cocke, T.C. Ho, J. Nanopart. Res. 2(7):2415-26 (2010)

[10] J. Selvi, S. Mahalakshmi, V. Parthasarathy, J. Inorg. Organomet. Polym. Mater. 27(6):1918-26 (2017)

[11] M. Aslam, M.A. Kalyar, Z.A. Raza, Appl. Phys. A 123(6):424 (2017)

[12] M. Aslam, M.A. Kalyar, Z.A. Raza, Mater. Res. Express 3(10), 105036 (2016)

[13] S.J. Hazarika, D. Mohanta, J. Mater. Res. 34(21):3666-77 (2019) 
[14] M. Nadeem, M. Ahmad, M.S. Akhtar, A. Shaari, S. Riaz, S. Naseem, M. Masood, M.A. Saeed, PloS one. 11(6):e0158084 (2016)

[15] S.B. Aziz, A.Q. Hassan, S.J. Mohammed, W.O. Karim, M. FZ. Kadir, H.A Tajuddin, N. NMY Chan, Nanomaterials, 9(2):216 (2019)

[16] S.B. Aziz, O.G. Abdullah, M.A. Rasheed, J. Appl. Polym. Sci. 134(21) (2017)

[17] M. Aslam, M.A. Kalyar, Z.A. Raza, J. Mater. Sci. Mater. Electron. 28(18) 13401-13 (2017)

[18] S.B. Aziz SB, H.M. Ahmed, A.M. Hussein, A.B. Fathulla, R.M. Wsw, R.T. Hussein, J. Mater. Sci. Mater. Electron. 26(10):8022-8 (2015)

[19] M. Aslam, M.A. Kalyar, Z.A. Raza, Polym. Bull. 78(3):1551-71 (2021)

[20] E.M. Antar, J. Radiat. Res. Appl. Sci. 7(1):129-34 (2014)

[21] K.S. Hemalatha, K. Rukmani, N. Suriyamurthy, B.M. Nagabhushana, Mater. Res. Bull. 51:438-46 (2014)

[22] S.B. Aziz SB, J. Electron. Mater. 45:736-745 (2016)

\section{Figures}




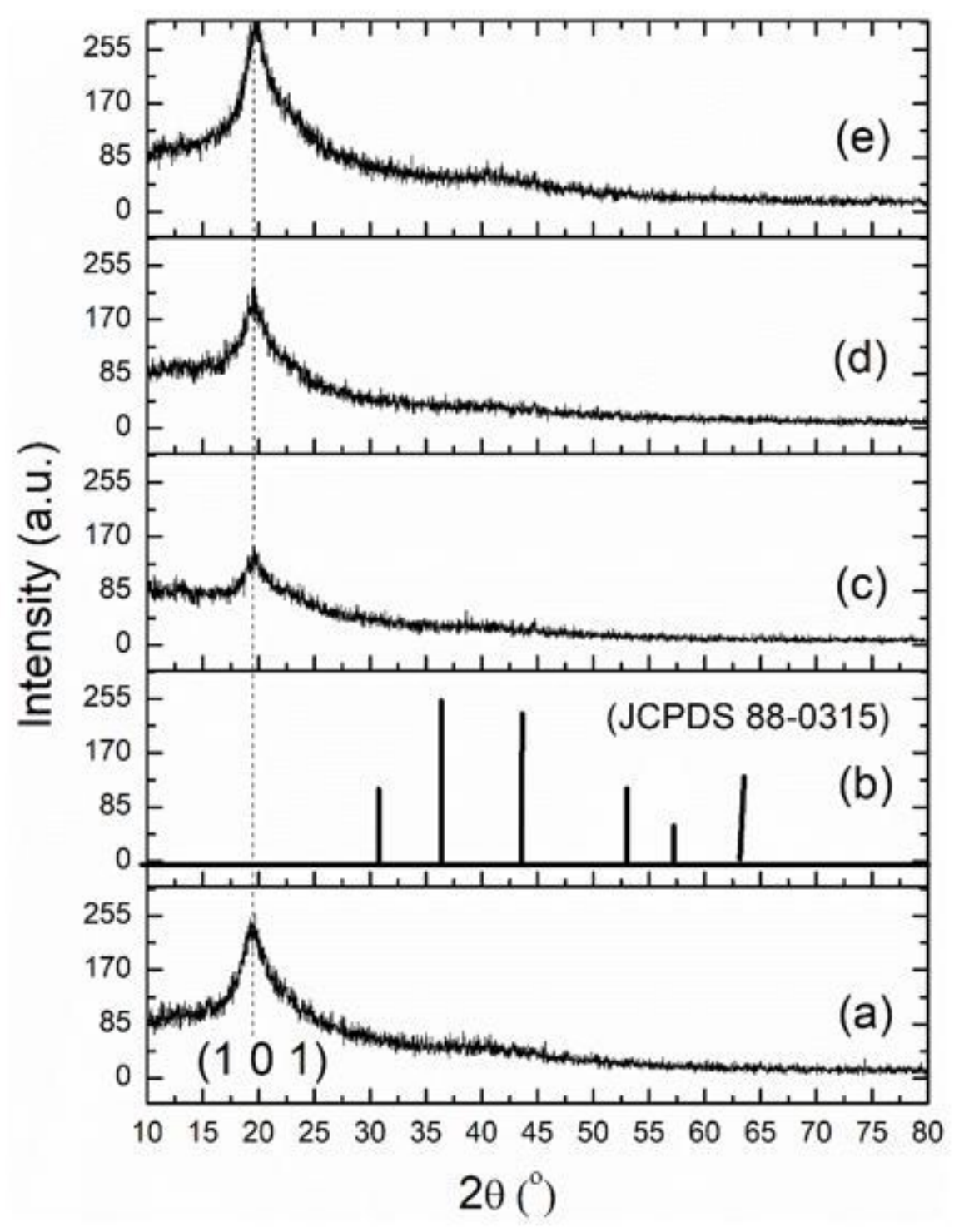

Figure 1

XRD patterns of (a) pure PVA, (b) pure $\mathrm{Fe}_{3} \mathrm{O}_{4}$, (c) $0.25 \mathrm{wt} \%$, (c) $0.5 \mathrm{wt} \%$ and (d) $1 \mathrm{wt} \% \mathrm{Fe}_{3} \mathrm{O}_{4}$ loaded PVA nanocomposites. 


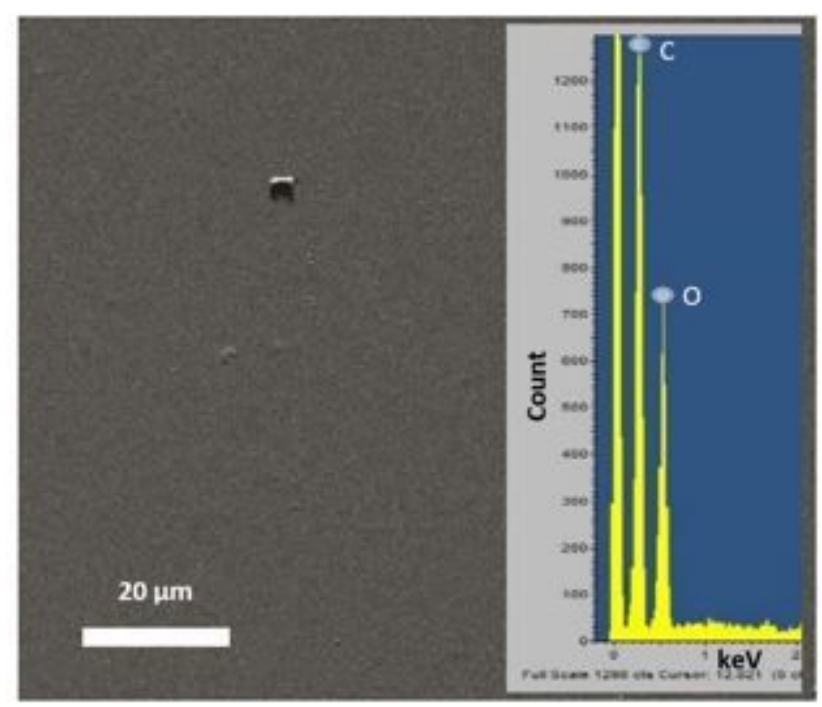

a

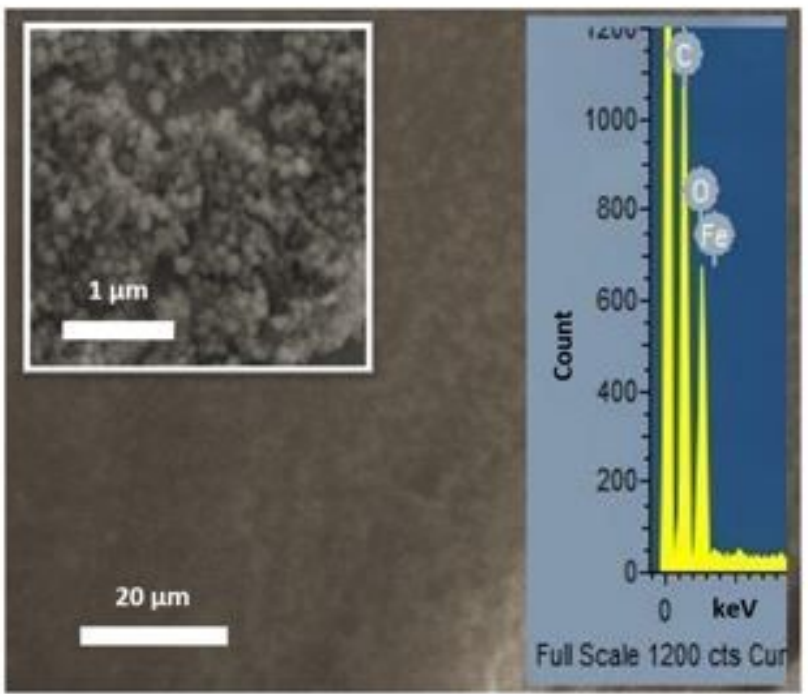

b

Figure 2

SEM micrographs of a) pure PVA and b) $\mathrm{Fe}_{3} \mathrm{O}_{4}$ loaded PVA nanocomposite films with their respective EDX spectra. 


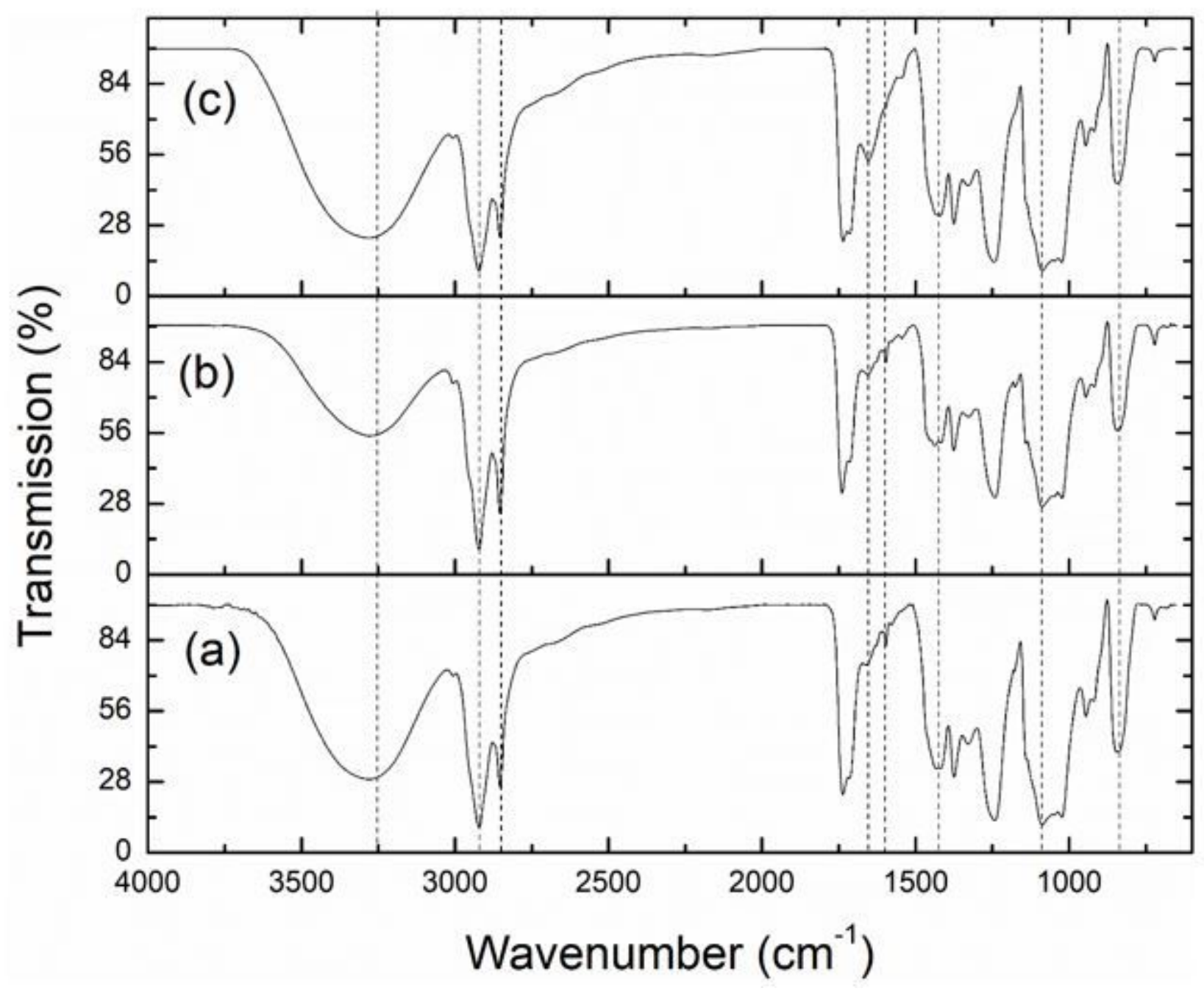

Figure 3

FTIR spectra of (a) pure PVA, (b) $0.5 \mathrm{wt} \%$ and (c) $1 \mathrm{wt} \% \mathrm{Fe}_{3} \mathrm{O}_{4}$ loaded PVA nanocomposites. 


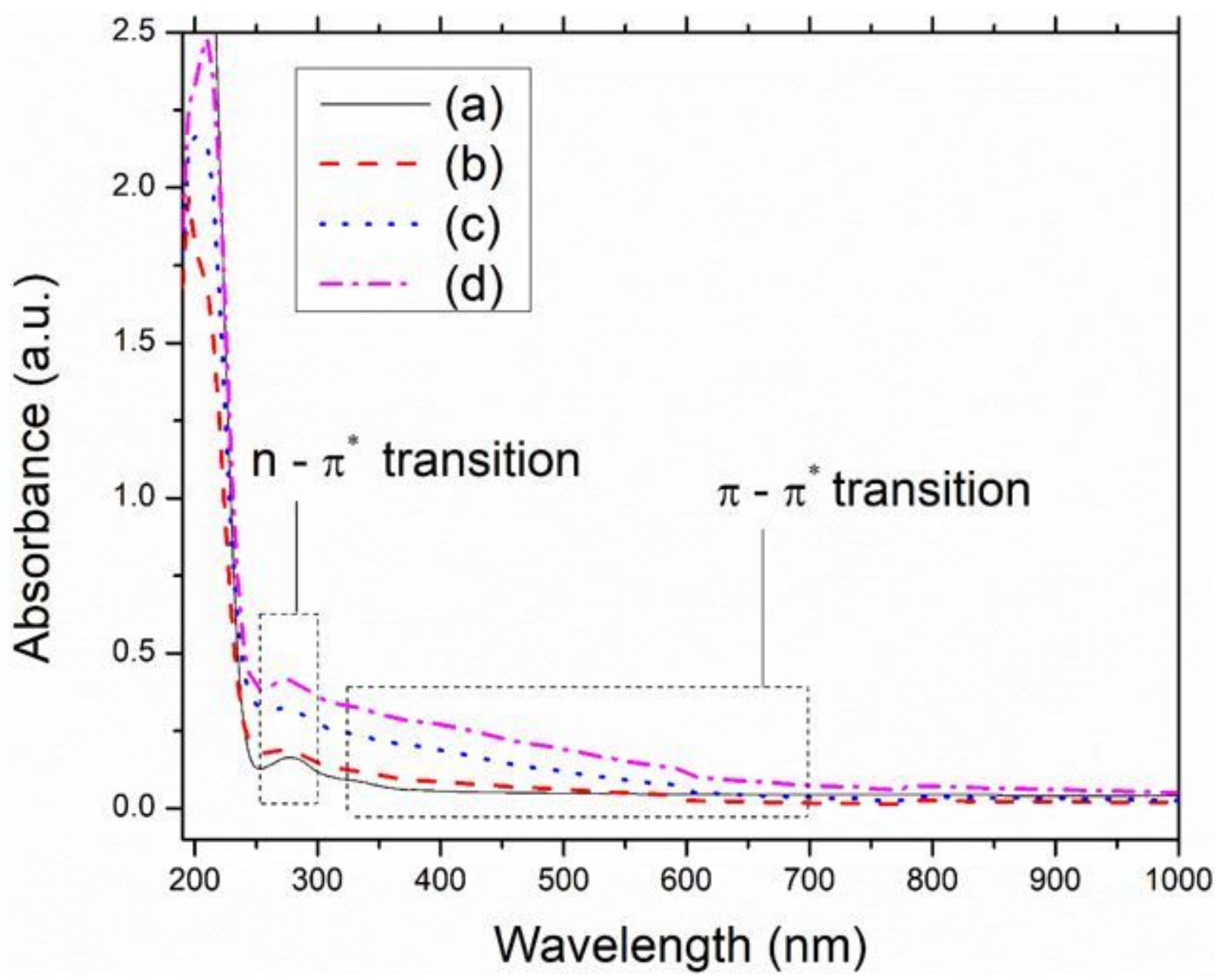

Figure 4

UV-vis absorbance spectra of (a) pure PVA, (b) $0.25 \mathrm{wt} \%$, (c) $0.5 \mathrm{wt} \%$ and (d) $1 \mathrm{wt} \% \mathrm{Fe}_{3} \mathrm{O}_{4}$ loaded PVA nanocomposites.
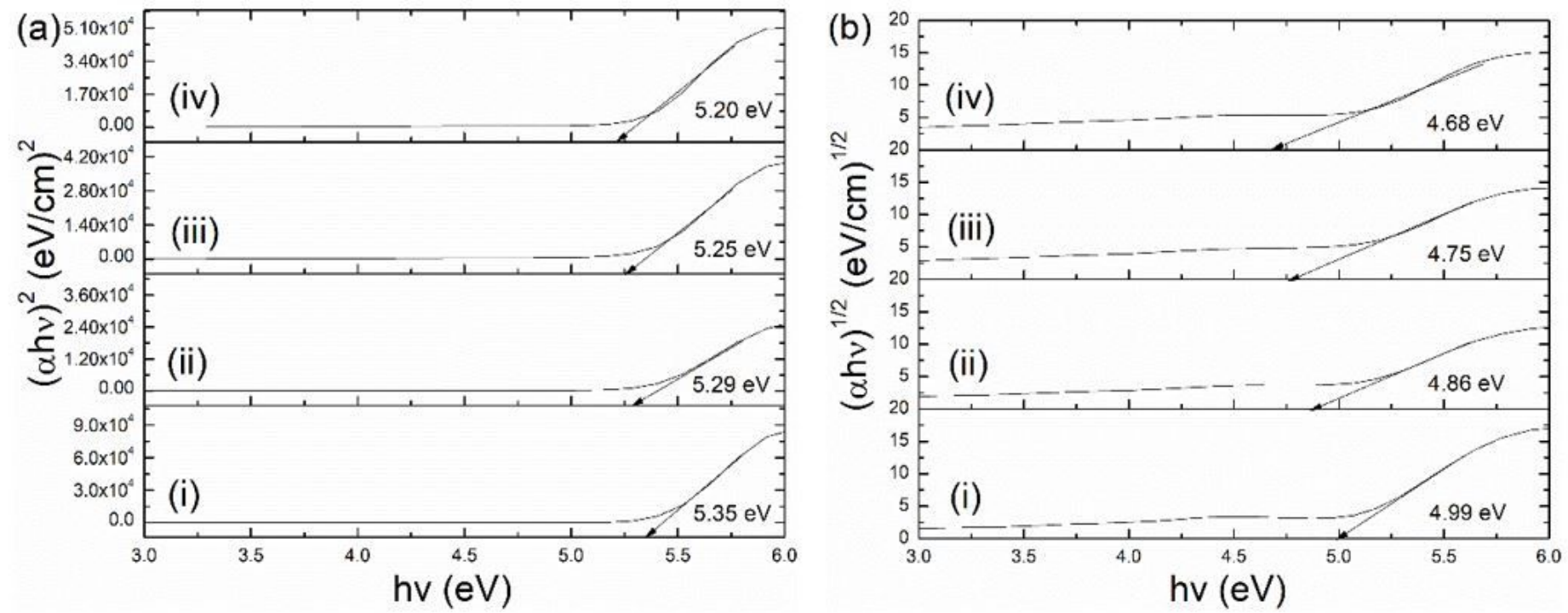
Figure 5

(a) Direct band gap versus energy plot and (b) indirect band gap versus energy plot for (i) pure PVA, (ii) 0.25 wt \%, (iii) 0.50 wt $\%$ and (1 wt\%) $\mathrm{Fe}_{3} \mathrm{O}_{4}$ loaded PVA nanocomposites.
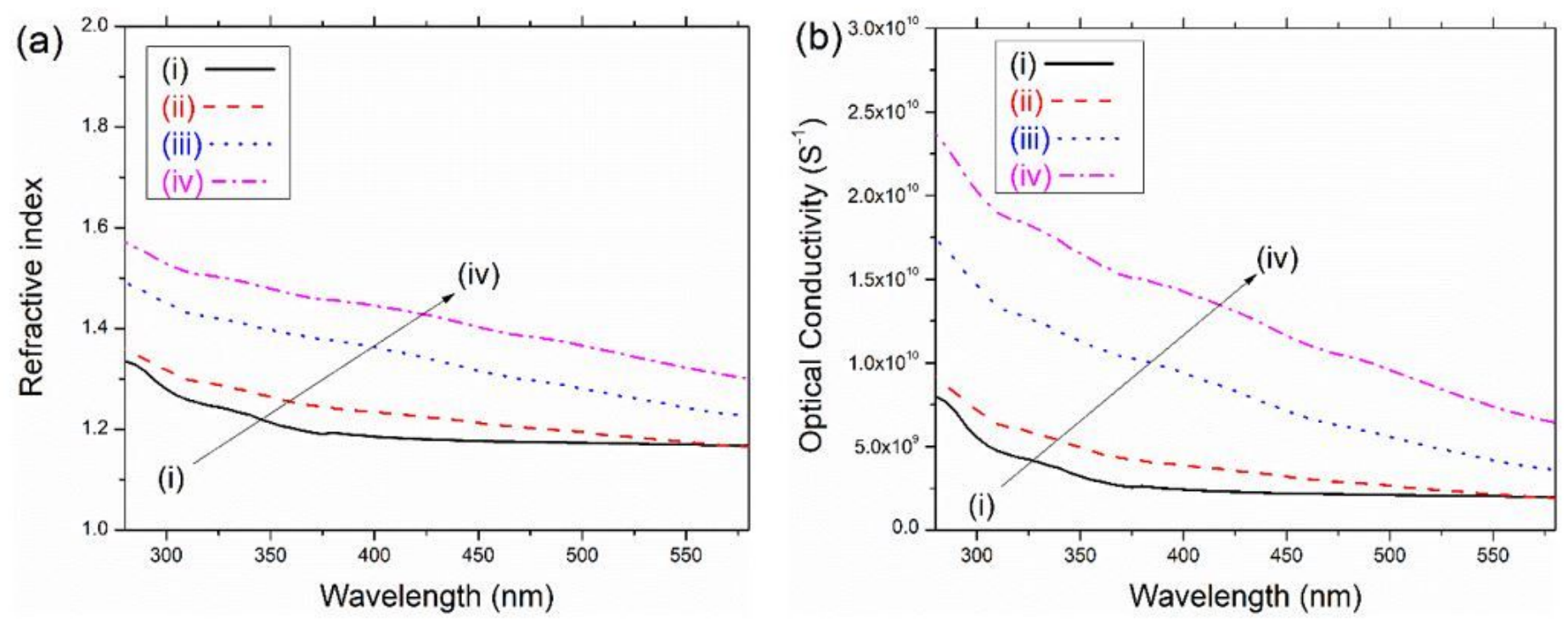

Figure 6

(a) Refractive index versus wavelength plot, and (b) optical conductivity versus wavelengths plot for (i) pure PVA, (ii) 0.25 wt\%, (iii) 0.50 wt\% and ( 1 wt\%) $\mathrm{Fe}_{3} \mathrm{O}_{4}$ loaded PVA nanocomposites. 